\title{
Coil Embolization for a Cerebral Aneurysm in a Heart Transplantation Patient: A Case Report
}

\author{
Masatoshi Takagaki, ${ }^{1}$ Tomoyoshi Nakagawa, ${ }^{1}$ Shuhei Kawabata, ${ }_{1}^{1}$ Nobuyuki Izutsu, \\ Takeo Nishida, ${ }^{1}$ Hajime Nakamura, ${ }^{1}$ and Haruhiko Kishima ${ }^{1}$
}

\begin{abstract}
The number of heart transplantations performed in Japan has been continuously increasing. Here, we report the case of a patient with an unruptured cerebral artery aneurysm after undergoing heart transplantation and was treated using coil embolization. The patient was a 50-yearold woman who was positive for heparin-induced thrombocytopenia (HIT) antibodies and underwent heart transplantation for dilated cardiomyopathy. An unruptured middle cerebral artery aneurysm was treated with coil embolization using argatroban as a heparin substitute. The patient was discharged without any complications. Despite these patients with heart transplantation are characterized by high HIT antibodies rate and the need for immunosuppressive agents, they currently have an excellent prognosis, especially in Japan. Therefore, the knowledge of patient characteristics after heart transplantation is essential for ensuring that these patients receive the most appropriate treatment.
\end{abstract}

Keywords: aneurysm, heparin, heart transplantation, thrombocytopenia, coil embolization

\section{Introduction}

The number of heart transplantations performed in Japan has been continuously increasing over recent years; with the 10 -year survival rate after heart transplantation reportedly reaching about $90 \%$, such patients can expect good prognosis. ${ }^{1)}$ Herein, we report the case of a patient with an unruptured cerebral artery aneurysm after undergoing heart transplantation and was treated using coil embolization.

\section{Case Presentation}

The patient was a 50-year-old female woman who was referred to our department for the treatment of an unruptured middle cerebral artery (MCA) aneurysm. On examination, no neurological deficits were detected. She was taking everolimus $1.75 \mathrm{mg} / \mathrm{day}$ and prednisolone $5 \mathrm{mg}$ /day as immunosuppressive agents. When the patient was 45 years ${ }^{1}$ Department of Neurosurgery, Osaka University Graduate School
of Medicine, Suita, Osaka, Japan

Received: May 13, 2019; Accepted: June 24, 2019

Online December 18, 2019

Copyright $(\subset 2020$ by The Japan Neurosurgical Society This work is licensed under a Creative Commons AttributionNonCommercial-NoDerivatives International License. old, cardiomegaly was observed at a routine medical examination, and she diagnosed with dilated cardiomyopathy. During the treatment for dilated cardiomyopathy, she was found to be positive for heparin-induced thrombocytopenia (HIT) antibodies; thus, heparin had not been used for her treatment since then. At the age of 47 years, she underwent implantation of a left ventricular assist device to maintain her heart function until heart transplantation. While she waited for the transplantation, head computed tomography (CT) revealed a localized subarachnoid hemorrhage (SAH) around the medulla oblongata. Head magnetic resonance (MR) angiography and CT angiography did not show any vascular diseases that might cause SAH; however, an unruptured aneurysm was detected in MCA (Fig. 1). Because of the patient's heart disease, the aneurysm was treated by conservative therapy at that time. The patient underwent heart transplantation at the age of 48 years. Immunosuppressive agents and aspirin $100 \mathrm{mg} /$ day were initiated after the procedure, and cilostazol $200 \mathrm{mg} / \mathrm{day}$ was added as a treatment for reversible cerebral vasoconstriction syndrome caused by the immunosuppressive agents.

The patient was referred to us 1 year after heart transplantation. Cerebral angiography revealed an aneurysm $5.1 \times 3.4$ $\mathrm{mm}$ in size with a bleb at the bifurcation of the right MCA (Fig. 2). After obtaining the patient's informed consent, she was scheduled for coil embolization for the MCA aneurysm. Our hospital's institutional review board approved the use of argatroban as a heparin substitute during the procedure.

Endovascular coil embolization was performed under general anesthesia. Prior to the procedure, an antibiotic was administered. A 7-Fr-long sheath was placed into the right femoral artery. Argatroban $6 \mathrm{mg}$ was injected intravenously, followed by a $20 \mathrm{mg} / \mathrm{h}$ continuous injection. The activated clotting time (ACT) was measured every $15 \mathrm{~min}$, and the infusion rate was controlled to maintain ACT for approximately 250 s. A 6-Fr guiding catheter (FUBUKI Hard MPR; ASAHI INTECC, Aichi, Japan) was placed in the right internal carotid artery. After performing angiography to determine the optimum working angle (Fig. 3A), a microcatheter (Excelsior SL 10; Stryker, Kalamazoo, MI, USA) was placed in the aneurysm. A balloon-assisted technique was then used to insert detachable coils (Target; Stryker) one after another into the aneurysm through the microcatheter. Angiography after coil embolization revealed the formation of a clot in the M2 superior trunk, so ozagrel sodium $40 \mathrm{mg}$ was drip-infused intravenously (Fig. 3B). During this, clot formation was also detected in the M2 inferior trunk (Fig. 3C). 

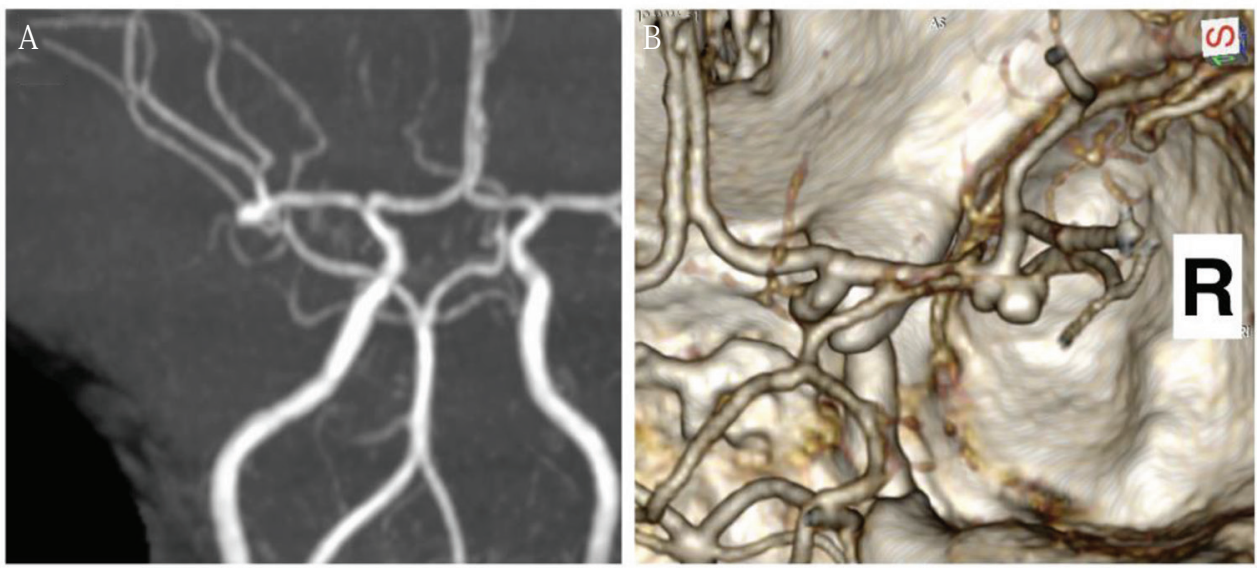

Fig. 1 Magnetic resonance angiography revealed an aneurysm of the right middle cerebral artery (A). Computed tomography angiography (B).
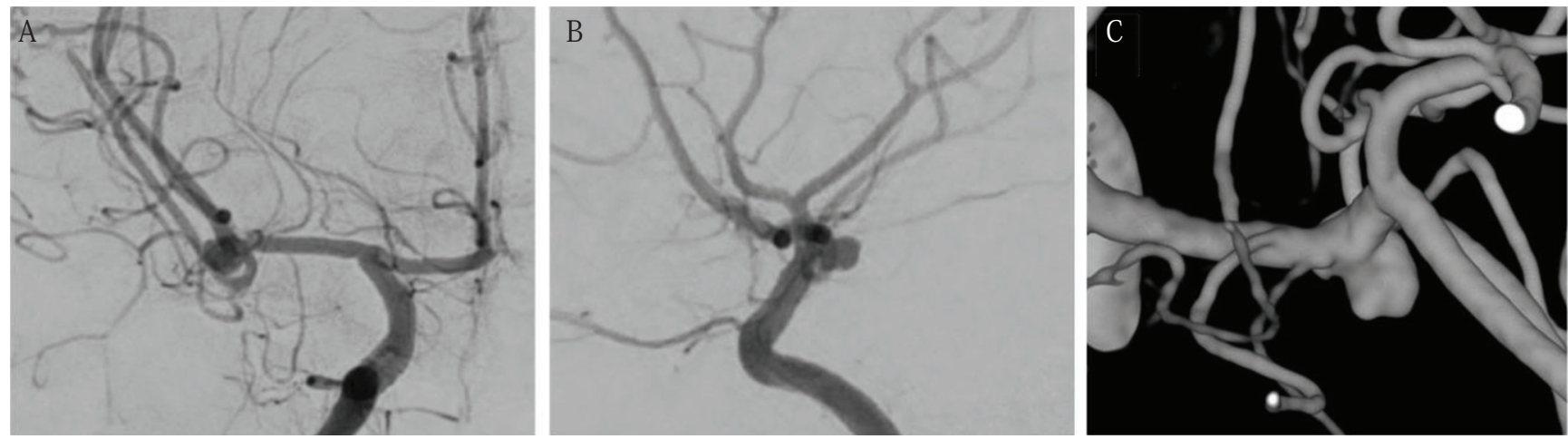

Fig. 2 Digital subtraction angiography (DSA; A: anteroposterior view, B: lateral view). Three-dimensional DSA showed a bleb on the aneurysm (C).
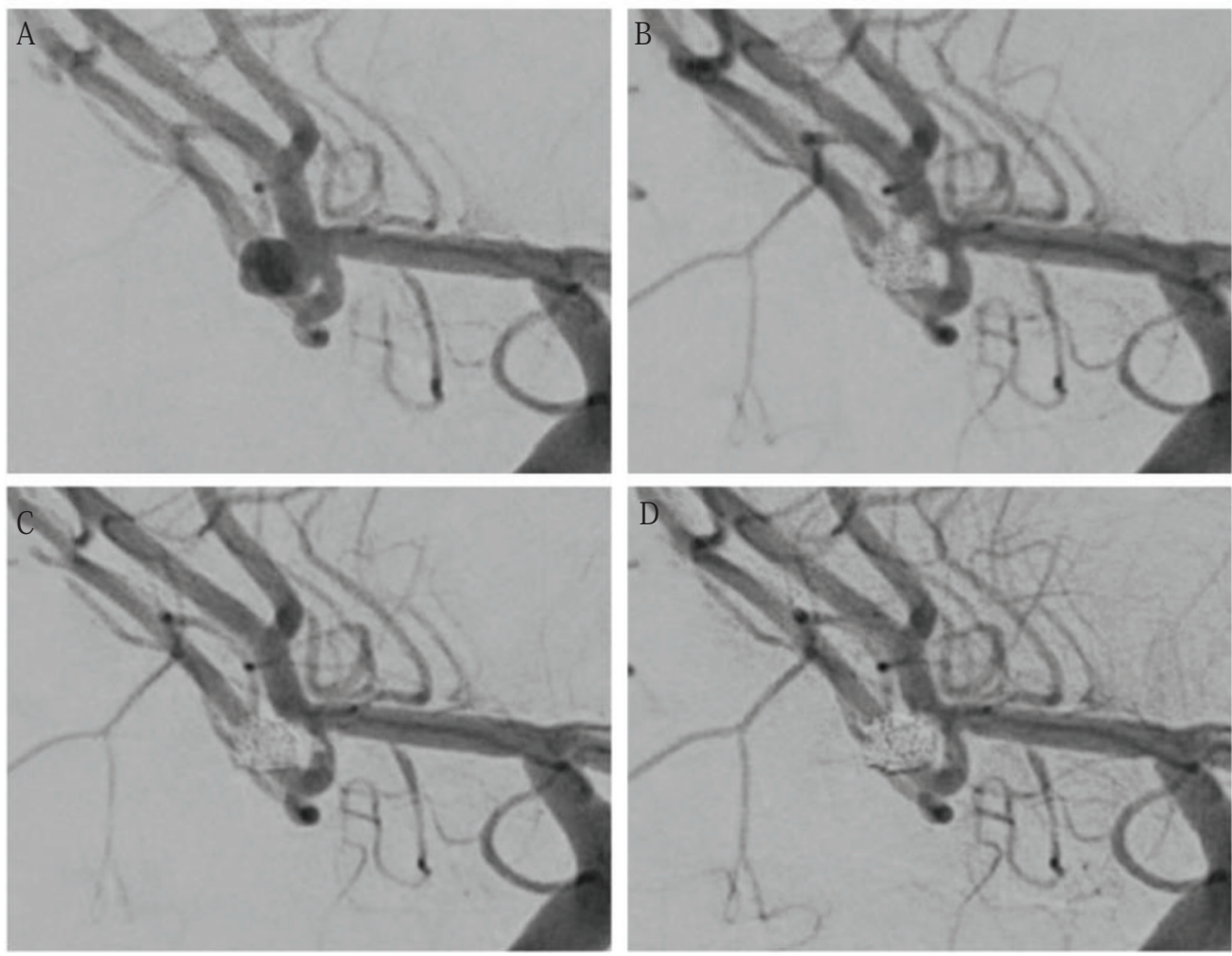

Fig. 3 Digital subtraction angiography before the coil embolization (A). Thrombosis was detected around the aneurysm neck (B and C). Final view (D). 
However, the final angiography confirmed the complete disappearance of the clots (Fig. 3D). After the procedure, argatroban $60 \mathrm{mg} /$ day was injected continuously for $48 \mathrm{~h}$. A head MR imaging acquired 1 day after the procedure revealed a non-symptomatic small infarction (Fig. 4A); however, the patient did not show any neurological symptom. Head MR angiography confirmed that there was no longer blood flow in the aneurysm (Fig. 4B). The patient's recovery was uneventful, with no complications, and she was discharged 9 days after the coil embolization.

\section{Discussion}

Ischemic stroke has been the most reported cerebrovascular complication in heart transplantation cases, at a rate of approximately $90 \%{ }^{2)}$ Furthermore, the occurrence of hemorrhagic stroke is unusual and that of subarachnoid hemorrhage remains unclear. ${ }^{2)}$ Moreover, there are no established guidelines for the treatment of unruptured aneurysms in heart transplantation patients. So far, the treatment of cerebral aneurysm with clipping or coiling in a heart transplantation patient has not been reported. This is the first report of coil embolization for a cerebral aneurysm in a heart transplant patient.

The first heart transplantation in Japan in accordance with the Organ Transplant Law of 1997 was performed in February 1999. Since the law was revised in 2010 , the number of heart transplantation has been continuously increasing. According to the Registry Report of Heart Transplantation in Japan, over 50 patients per year undergo the procedure. ${ }^{1)}$ The cumulative total by August 2018 was more than 400 heart transplantations, so it can be expected that it will become less rare to encounter patients after heart transplantation with unruptured cerebral aneurysms. Determining the strategy for such cases will require neurosurgeons who are not familiar with transplantation medicine to have knowledge about the current status and prognosis of heart transplantation.

The survival rate after heart transplantation in Japan is now excellent, with a 5-year survival rate of $92.7 \%$ and a 10 -year survival rate of $89.6 \%$. $^{1)}$ Indeed, the 10 -year survival rate compares favorably with that of the United States, reported to be
$53 \%$.) The present patient was only 50 years old, the postoperative course was good, and her general condition was stable, so she could be expected to have a good long-term prognosis. It was therefore decided, with the patient's consent, to perform the embolization to prevent rupture of the aneurysm.

An important reason for the improvement in the prognosis of transplantation patients has been the developments in immunosuppressive agents. The administration of immunosuppressants is essential after organ transplantation. Potent immunosuppressants are especially necessary early after the transplantation; after that, the immunosuppressive agents are gradually reduced, but they cannot be discontinued. Patients taking immunosuppressants after organ transplantation are at risk of perioperative infection, but there is no concensus on the appropriate approach to be followed. ${ }^{3)}$ Kurata et al. reported one infectious complication in three cases of abdominal surgery after kidney transplantation. They considered immunosuppressants as the cause, and suggested that careful care is needed to prevent perioperative infection. ${ }^{4)}$ There have been few reports about neurosurgery after organ transplantation. Hokari et al. ${ }^{5)}$ reported that 11 patients on immunosuppressant treatment had undergone cerebral aneurysm clipping, with good results. They reported that, although the risk of perioperative infection was no higher for these patients, the consequences of infection would be serious. The risk of infection therefore requires sufficient attention, and less invasive endovascular treatment should be adequate for these patients. ${ }^{5)}$

It is known that stenosis of coronary arteries progresses after heart transplantation. ${ }^{\text {) }}$ In the present case, an antiplatelet agent was administered to prevent coronary artery stenosis. Cilostazol was also prescribed because of the patient's history of reversible cerebral vasoconstriction syndrome, thought to be caused by the immunosuppressants. In addition to the risk of infectious complications resulting from immunosuppressant usage, there was also the risk of hemorrhagic complications resulting from the administration of antiplatelet agents. Until recently, clipping was considered the best treatment for an MCA aneurysm. ${ }^{7}$ However, a recent report suggested that the results of endovascular treatment are equivalent to those of
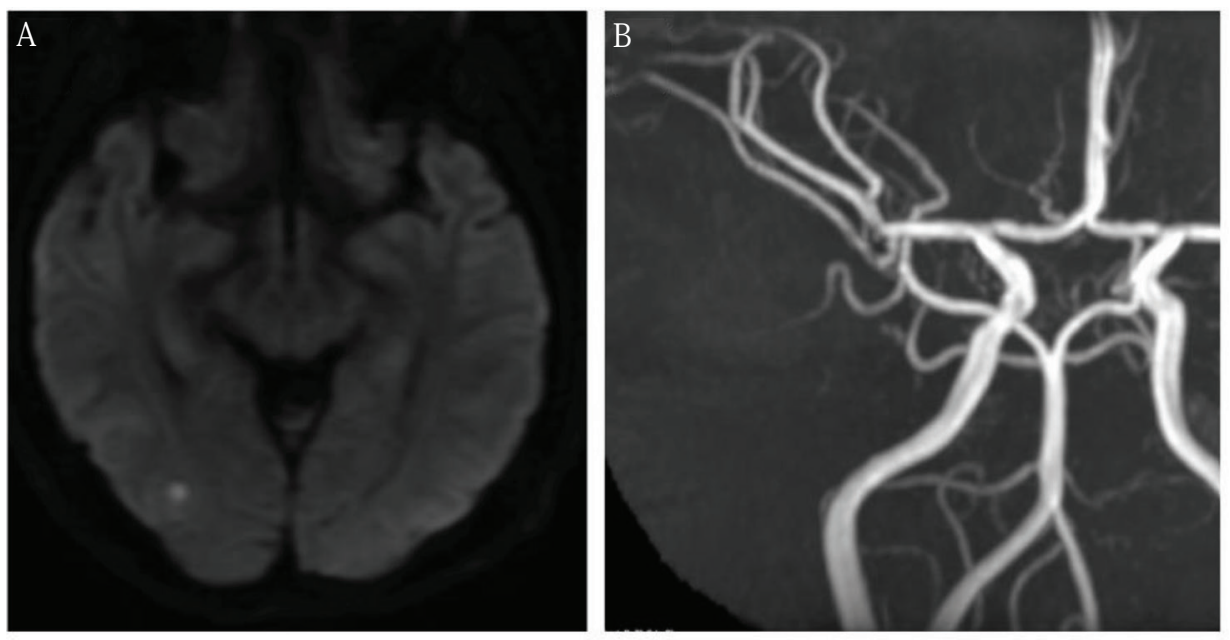

Fig. 4 Diffusion-weighted imaging after the coil embolization revealed a small infarction in the right hemisphere (A). Magnetic resonance angiography confirmed there was no blood flow in the aneurysm (B). 
clipping. ${ }^{8)}$ Although the present case involved an MCA aneurysm, we considered endovascular treatment to be preferable to clipping. Moreover, coronary intervention after heart transplantation has been reported. ${ }^{9)}$ Therefore, we considered that neurointervention was also properly performed. However, due to immunosuppressant usage, care should be taken to avoid infectious complications, even in neurointervention cases; therefore, an antibiotic was used in the perioperative period. The patient had a history of HIT, which can cause problems in performing an endovascular treatment. However, substitution of heparin with argatroban overcame the problem and we were able to obtain good results with endovascular treatment.

The patient underwent repeated coronary angiography because of her heart disease, and heparin was frequently administered, which may have caused the HIT. It has been reported that $20-40 \%$ of patients waiting for heart transplantation or after heart transplantation are positive for HIT antibodies. ${ }^{10,11)}$ In the present case, heparin was used repeatedly from the early stage of the dilated cardiomyopathy diagnosis until the HIT antibodies were detected. Guidelines for coronary endovascular treatment recommend argatroban as an alternative drug to heparin during endovascular treatment for patients with HIT, ${ }^{12)}$ and this now has insurance coverage in Japan. There is no clear guideline yet about neuroendovascular treatment for patients with a history of HIT, but there is a report that neuroendovascular treatment is performed using argatroban as a substitute drug. ${ }^{13)}$ However, the problem with the use of argatroban is that a large amount is required to maintain the ACT at the desired level; and, even when the ACT is kept at the appropriate level, thrombus formation may still occur, as in the present case. Evaluating clotting ability under the administration of argatroban requires the measurement of the activated partial thromboplastin time, but it is difficult to obtain the results of this immediately. It may therefore be necessary to promptly administer ozagrel sodium and other appropriate treatment when thrombus formation is observed.

\section{Conclusion}

Patients who undergo heart transplantation now have an excellent prognosis. These patients are characterized by high HIT antibody rate and the need for immunosuppressive agents. Therefore, the knowledge of patient characteristics after heart transplantation is essential for ensuring that these patients receive the most appropriate treatment.

\section{Conflicts of Interest Disclosure}

All authors report no conflicts of interest regarding this article.

\section{References}

1) Fukushima N, Ono M, Saiki Y, Sawa $Y$, Nunoda S, Isobe M: Registry report on heart transplantation in Japan (June 2016). Circ J 81: 298-303, 2017

2) Alejaldre A, Delgado-Mederos R, Santos MÁ, Martí-Fàbregas J: Cerebrovascular complications after heart transplantation. Curr Cardiol Rev 6: 214-217, 2010

3) Ota T, Rocha R, Wei LM, Toyoda Y, Gleason TG, Bermudez C: Surgical outcomes after cardiac surgery in liver transplant recipients. J Thorac Cardiovasc Surg 145: 1072-1076, 2013

4) Kurata N, Hachisuka T, Tochigi K, Shikano T, Hashimoto Y, Mori T: Digestive system neoplasms following kidney transplantation: an evaluation of three cases. Jpn J Gastroenterol Surg 49: 569-577, 2016

5) Hokari M, Nakayama N, Kazumata K, et al.: Surgical Outcome of cerebral aneurysm clipping treated with immunosuppressants: report of 11 cases and review of the literature. Neurol Med Chir (Tokyo) 57: 122-127, 2017

6) Christie JD, Edwards LB, Aurora P, et al.: Registry of the International Society for Heart and Lung Transplantation: twenty-fifth official adult lung and heart/lung transplantation report-2008. J Heart Lung Transplant 27: 957-969, 2008

7) Smith TR, Cote DJ, Dasenbrock HH, et al.: Comparison of the efficacy and safety of endovascular coiling versus microsurgical clipping for unruptured middle cerebral artery aneurysms: a systematic review and meta-analysis. World Neurosurg 84: 942-953, 2015

8) Ruan C, Long H, Sun H, et al.: Endovascular coiling vs. surgical clipping for unruptured intracranial aneurysm: a meta-analysis. $\mathrm{Br} \mathrm{J} \mathrm{Neu-}$ rosurg 29: 485-492, 2015

9) Tsuji M, Nitta D, Minatsuki S, et al.: Emergency percutaneous coronary intervention for left main trunk thrombus following orthotopic heart transplantation. ESC Heart Fail 6: 575-578, 2019

10) Hourigan LA, Walters DL, Keck SA, Dec GW: Heparin-induced thrombocytopenia: a common complication in cardiac transplant recipients. J Heart Lung Transplant 21: 1283-1289, 2002

11) Schroder JN, Daneshmand MA, Villamizar NR, et al.: Heparininduced thrombocytopenia in left ventricular assist device bridge-totransplant patients. Ann Thorac Surg 84: 841-845; discussion 845-846, 2007

12) Lewis BE, Matthai WH, Cohen M, et al.: Argatroban anticoagulation during percutaneous coronary intervention in patients with heparin-induced thrombocytopenia. Catheter Cardiovasc Interv 57: 177-184, 2002

13) Kim S, Ohtaki M, Tsuda H, Kimura Y: A case of carotid artery stenting following heparin-induced thrombocytopenia development. $\mathrm{J} \mathrm{Neu-}$ roendovasc Ther 9: 305-309, 2015 